\title{
AN EXTREMAL PROBLEM FOR THE GEOMETRIC MEAN OF POLYNOMIALS
}

\author{
E. BELLER AND D. J. NEWMAN ${ }^{1}$
}

ABstract. Let $M_{0, n}$ be the maximum of the geometric mean of all $n$th degree polynomials $\sum^{n} a_{k} e^{i k t}$ which satisfy $\left|a_{k}\right|=1$, $k=0,1, \cdots, n$. We show the existence of certain polynomials $R_{n}$ whose geometric mean is asymptotic to $\sqrt{ } n$, thus proving that $\boldsymbol{M}_{\mathbf{0}, n}$ is itself asymptotic to $\sqrt{ } \boldsymbol{n}$.

Consider the class $\mathscr{P}_{n}$ of all $n$th degree polynomials $\sum_{k=0}^{n} a_{k} z^{k}$ for which $\left|a_{k}\right|=1, k=0,1, \cdots, n$. Following the usual notation, let

$$
M_{r}(f)=\left((2 \pi)^{-1} \int_{-\pi}^{\pi}\left|f\left(e^{i \theta}\right)\right|^{r} d \theta\right)^{1 / r}
$$

for $r>0$, and let

$$
M_{0}(f)=G(f)=\exp \left((2 \pi)^{-1} \int_{-\pi}^{\pi} \log \left|f\left(e^{i \theta}\right)\right| d \theta\right),
$$

so that, in particular, for $r=0,1$, and $2, M_{r}(f)$ is the geometric mean, arithmetic mean, and mean square of $f$, respectively.

Now, for $f \in \mathscr{P}_{n}$, and $0 \leqq r<2$, we have $M_{r}(f) \leqq M_{2}(f)=(n+1)^{1 / 2}$. The question arises, how close can $M_{r}(f)$ be to $(n+1)^{1 / 2}$, the mean square? To formulate our question in more precise terms, let

$$
\boldsymbol{M}_{r, n}=\max _{\{f\}} M_{r}(f) \quad\left(f \in \mathscr{P}_{n}\right)
$$

for $r<2$, so that $\boldsymbol{M}_{r, n} \leqq(n+1)^{1 / 2}$. For each $r<2$, we now ask: Is $\boldsymbol{M}_{r, n}$ asymptotic to $\sqrt{ } n$ as $n \rightarrow \infty$ ?

Newman [3] constructed $P_{n} \in \mathscr{P}_{n}$ for which $M_{4}^{4}\left(P_{n}\right)=n^{2}+O\left(n^{3 / 2}\right)$, and thereby proved that $M_{1}\left(P_{n}\right)>\sqrt{ } n-c$. Beller [1] noted that this

Received by the editors May 22, 1972 and, in revised form, October 9, 1972.

AMS (MOS) subject classifications (1970). Primary 30A06, 42A04; Secondary 30A08.

Key words and phrases. Extremal polynomials, coefficients of constant modulus, geometric mean, asymptotic to mean square, zeros of polynomials, reverse arithmeticgeometric inequality.

${ }^{1}$ Research of second author partially supported by Grant AF 69-1736.

${ }^{2}$ J. E. Littlewood [2], working with similar polynomials $F_{n}$, obtained a sharper estimate for $M_{4}^{4}\left(F_{n}\right)$, namely, $M_{4}^{4}\left(F_{n}\right)=n^{2}+k n^{3 / 2}+O(n)$, where $k=\sqrt{ } 2-(2 / \pi)(\sqrt{ } 2-1)$.

(c) American Mathematical Society 1973 
implies the following, more complete result: $\boldsymbol{M}_{r, n}>\sqrt{ } n-c / r$, for $0<r<2$ ( $c$ an absolute constant), thus answering our question affirmatively for all $r<2$ except $r=0$.

It is the purpose of this paper to show that $\boldsymbol{M}_{0, n} \sim \sqrt{ } n$. It may be noted that this result yields an interesting corollary concerning the zeros of polynomials. Since, for all $f \in \mathscr{P}_{n}$, the product of all the zeros of $f(z)$ is numerically equal to 1 , it follows from Jensen's theorem that $G(f)=$ $\prod_{i}\left|r_{i}\right|$, where the $r_{i}$ are the zeros of $f(z)$ for which $\left|r_{i}\right|>1$. Thus, our result can be rephrased as follows: The maximum - taken over all $f \in \mathscr{P}_{n}-$ of the product of the moduli of the zeros of $f(z)$ outside the unit circle, is asymptotic to $\sqrt{ } n$.

Before proceeding with our theorem, let us review the situation for $r>2$. Since, for such $r, M_{r}(f) \geqq(n+1)^{1 / 2}$, we consider

$$
\boldsymbol{m}_{r, n}=\min _{\{f\}} M_{r}(f) \quad\left(f \in \mathscr{P}_{n}\right) .
$$

We also define $M_{\infty}(f)=\sup _{\{\theta\}}\left|f\left(e^{i \theta}\right)\right|$. Beller [1] has shown that $\boldsymbol{m}_{r, n}$ is asymptotic to $\sqrt{ } n$, for $2<r<\infty$. This, taken together with our present result, gives us an affirmative answer to our question for all $r \geqq 0$, except $r=\infty$, which still remains unsettled.

We now state our

THEOREM. $\boldsymbol{M}_{0, n} \sim \sqrt{ } n$. In fact, $\boldsymbol{M}_{0, n}>\sqrt{ } n-c \log n$, where $c$ is an absolute constant.

Proof. Choose $P_{n} \in \mathscr{P}_{n}$ which satisfy

$$
M_{4}\left(P_{n}\right)=\sqrt{ } n+O(1),
$$

e.g., the $P_{n}$ which Newman [3] constructed. Now, in estimating $G\left(P_{n}\right)$, one encounters difficulty where $\left|P_{n}\right|$ is close to zero. We will show that, by appropriately changing the constant term of $P_{n}$, this difficulty is eliminated.

Let $f^{+}(x)=f(x) \quad(f(x) \geqq 0) ; f^{+}(x)=0 \quad(f(x)<0)$. We will need the following well-known inequality:

$$
(2 \pi)^{-1} \int_{-\pi}^{\pi} \log \left|e^{i t}+\alpha\right| d t=\log ^{+}|\alpha|,
$$

where $\alpha$ is any complex number.

Now, let $Q_{n}(z)=P_{n}(z)-a_{0}$, where $a_{0}$ is the constant term of $P_{n}(z)$. By reversing the order of integration and applying (2), we find that

$$
\frac{1}{2 \pi} \int_{-\pi}^{\pi}\left(\frac{1}{2 \pi} \int_{-\pi}^{\pi} \log \left|e^{i t}+Q_{n}\left(e^{i \theta}\right)\right| d \theta\right) d t=\frac{1}{2 \pi} \int_{-\pi}^{\pi} \log ^{+}\left|Q_{n}\left(e^{i \theta}\right)\right| d \theta .
$$


Thus, by the First Mean Value Theorem, there exists a real $\delta$ such that

$$
(2 \pi)^{-1} \int_{-\pi}^{\pi} \log \left|e^{i \delta}+Q_{n}\left(e^{i \theta}\right)\right| d \theta=(2 \pi)^{-1} \int_{-\pi}^{\pi} \log ^{+}\left|Q_{n}\left(e^{i \theta}\right)\right| d \theta .
$$

Let $R_{n}(z)=e^{i \delta}+Q_{n}(z)$, so that $R_{n} \in \mathscr{P}_{n}$. Our Theorem now follows directly from the following

Proposition. $G\left(R_{n}\right)>\sqrt{ } n-c \log n$, where $c$ is an absolute constant.

Proof of Proposition. By Minkowski's inequality we have $M_{4}\left(P_{n}\right)-1$ $\leqq M_{4}\left(Q_{n}\right) \leqq M_{4}\left(P_{n}\right)+1$, and this, combined with (1), yields

$$
(2 \pi)^{-1} \int_{-\pi}^{\pi}\left|Q_{n}\right|^{4} d \theta=n^{2}+O\left(n^{3 / 2}\right) .
$$

We also have

$$
(2 \pi)^{-1} \int_{-\pi}^{\pi}\left|Q_{n}\right|^{2} d \theta=n .
$$

We now need the following lemma, which is actually a reverse arithmetic-geometric inequality:

LEMMA 1. If $f(x) \geqq 0, \int_{a}^{b} f(x) d x=\lambda>0$, and $\int_{a}^{b} f^{2}(x) d x=\mu$, then

$$
\int_{a}^{b} \log (1+f(x)) d x \geqq\left(\lambda^{2} / \mu\right) \log (1+\mu / \lambda) .
$$

Proof. Set $p(x)=(1 / \lambda) f(x)$, so that $p(x)$ is a nonnegative weight function. Let $\mu / \lambda=m$, so that $\int_{a}^{b} f(x) p(x) d x=m$. In these terms, we want to show that

$$
\int_{a}^{b} \frac{\log (1+f(x))}{f(x)} p(x) d x \geqq(1 / m) \log (1+m) .
$$

But direct differentiation shows that $\phi(t)=(1 / t) \log (1+t)$ is convex on $[0, \infty)$, so that we may apply Jensen's inequality (see Zygmund [4, Vol. I, p. 24]), namely,

$$
\int_{a}^{b} \phi(f(x)) p(x) d x \geqq \phi\left(\int_{a}^{b} f(x) p(x) d x\right) . \quad \text { Q.E.D. }
$$

Remark. Since the function $\left(\lambda^{2} / \mu\right) \log (1+\mu / \lambda)$ is increasing in $\lambda$, for $\lambda>0$, and decreasing in $\mu$, the conclusion of Lemma 1 still holds under the weaker hypothesis $f(x) \geqq 0, \int f(x) d x \geqq \lambda>0, \int f^{2}(x) d x \leqq \mu$. 
LEMmA 2. If $\int_{0}^{1} F(x) d x \geqq A>1$ and $\int_{0}^{1} F^{2}(x) d x \leqq B$, then ${ }^{3}$

$$
\int_{0}^{1} \log ^{+} F(x) d x \geqq \frac{(A-1)^{2}}{B-2 A+1} \log \left(\frac{B-A}{A-1}\right) .
$$

ProOF. We apply our previous remark to $f(x)=(F(x)-1)^{+}$, so that

and

$$
\int_{0}^{1} f(x) d x \geqq \int_{0}^{1}(F(x)-1) d x \geqq A-1>0
$$

$$
\int_{0}^{1} f^{2}(x) d x \leqq \int_{0}^{1}(F(x)-1)^{2} d x \leqq B-2 A+1,
$$

and we conclude that

$$
\int_{0}^{1} \log ^{+} F(x) d x=\int_{0}^{1} \log (1+f(x)) d x \geqq \frac{(A-1)^{2}}{B-2 A+1} \log \left(\frac{B-A}{A-1}\right),
$$

as required.

Now let $F(x)=\left|Q_{n}\left(e^{2 \pi i x}\right)\right|^{2}$. In terms of $F,(4)$ and (5) become

$$
\int_{0}^{1} F(x) d x=n ; \quad \int_{0}^{1} F^{2}(x) d x \leqq n^{2}+c_{1} n^{3 / 2},
$$

where $c_{1}$ is some absolute constant. Applying Lemma 2, we have

$$
\begin{aligned}
(2 \pi)^{-1} \int_{-\pi}^{\pi} \log ^{+}\left|Q_{n}\left(e^{i \theta}\right)\right| d \theta & =\frac{1}{2} \int_{0}^{1} \log ^{+} F(x) d x \\
> & \frac{1}{2}\left(\frac{1-2 / n}{1+c_{1} / \sqrt{ } n}\right) \log n>\frac{1}{2} \log n-\left(\frac{c}{\sqrt{ } n}\right) \log n,
\end{aligned}
$$

where $c$ is another constant.

Combining (3) and (6), we conclude that

$$
\begin{aligned}
G\left(R_{n}\right) & =\exp \left((2 \pi)^{-1} \int_{-\pi}^{\pi} \log ^{+}\left|Q_{n}\left(e^{i \theta}\right)\right| d \theta\right) \\
& \geqq \sqrt{ } n \exp (-(c / \sqrt{ } n) \log n)>\sqrt{ } n-c \log n,
\end{aligned}
$$

thus proving the Proposition.

\section{REFERENCES}

1. E. Beller, Polynomial extremal problems in $L^{p}$, Proc. Amer. Math. Soc. 30 (1971), 249-259. MR 43 \#7598.

8 It can be shown that this inequality is the best possible. 
2. J. E. Littlewood, On polynomials $\sum^{n} \pm z^{m}, \sum^{n} e^{\alpha} m^{i} z^{m}, z=e^{\theta i}$, J. London Math. Soc. 41 (1966), 367-376. MR 33 \#4237.

3. D. J. Newman, An $L^{1}$ extremal problem for polynomials, Proc. Amer. Math. Soc. 16 (1965), 1287-1290. MR 32 \#2589.

4. A. Zygmund, Trigonometric series, 2nd ed., Cambridge Univ. Press, New York, 1959. MR 21 \#6498.

Department of Mathematics, Newark College of Engineering, Newark, New JERSEY 07102

Department of Mathematics, Yeshiva University, New York, New York 10033 (Current address of D. J. Newman)

Current address (E. Beller): Department of Mathematics, Bar-Ilan University, Ramat Gan, Israel 